\title{
Flight in a Packing of Disks
}

\section{Fejes Tóth}

Mathematical Institute of the Hungarian Academy of Sciences,

P.O. Box 127, H-1364 Budapest, Hungary

With sincere friendship dedicated to August Florian

\begin{abstract}
For a packing in the plane consisting of open convex domains, none of which contains the origin $O$, let $\lambda(r)$ be the length of the shortest path which evades the domains and connects $O$ with a point at a distance $r$ from $O$. Upper and lower bounds are given for the supremum of $\lambda(r)$ taken over all packings whose members are domains of diameter at least 2 .
\end{abstract}

\section{Introduction}

By a packing of disks we mean a set of pairwise disjoint open convex domains. Let $F$ be a family of disks. We consider a packing of disks from $F$ none of which contains the origin $O$. Let $\lambda(r)$ be the length of a shortest path which evades the disks and connects $O$ with a point of the circle of radius $r$ centered at $O$. Let $\Lambda(F, r)$ be the supremum of $\lambda(r)$ extended over all packings defined above.

In this paper we consider the case when $F$ consists of all convex domains of diameter at least 2 . In Section 1 we give an upper bound and in Section 2 a lower bound for $\Lambda(r)=\Lambda(F, r)$. Section 3 contains some remarks.

\section{Upper Bound}

We claim that there is a constant $c$ such that, for $r \geq 2$,

$$
\Lambda(r) \leq r^{2}+\frac{1}{2} \ln r+c
$$

We say that a boundary point $P$ of a disk $d$ is on the arrival side or on the departure 
side of $d$ according to whether the elongation of the segment $O P$ does or does not intersect $d$.

Let $P$ move on bd $d$ into a new position $P^{\prime}$. We write $\Delta r=\overline{O P^{\prime}}-\overline{O P}$ and $\Delta \lambda$ for the length of the arc $P P^{\prime}$. The proof of (1) is based on the following.

Lemma. Let $P$ be a point on the arrival side of a disk $d$ of diameter at least 2 . Assuming that $\overline{O P}=r \geq 2$, there is a point $P^{\prime}$ on the departure side of $d$ for which

$$
\frac{\Delta r}{\Delta \lambda} \geq \sqrt{1+r^{2}}-r
$$

Proof. Let $A B$ be a segment of length 2 containing $P$. We write $\overline{A P}=a, \overline{B P}=b$, $\angle O P A=\varphi+90^{\circ}$, and $\sqrt{1+r^{2}}-r=\delta=\delta(r)$. Let $A B$ be in a position such that $(\overline{O A}-r) / a=\delta$. Then

$$
\overline{O A}^{2}=r^{2}+a^{2}+2 r a \sin \varphi=(r+a \delta)^{2},
$$

whence, using the identity $\left(1-\delta^{2}\right) / 2 r=\delta$, we obtain

$$
\sin \varphi=(1-a) \delta
$$

Applying this formula for $-\varphi$ instead of $\varphi$, we have $-\sin \varphi=(1-b) \delta$ which shows that we have automatically $(\overline{O B}-r) / b=\delta$.

Let $\psi$ be the obtuse or right angle made by a support line $s$ of $d$ through $P$ and the line $P O$. Assuming that $\psi \geq 90^{\circ}+\operatorname{arc} \sin \delta$, (1.2) implies that all points $P^{\prime}$ on the arrival side of $d$ which arise by continuously increasing $\Delta r$ satisfy (1.1). So does $P^{\prime}$ in the limiting case when $O P^{\prime}$ touches $d$, proving the lemma in the case $\psi \geq 90^{\circ}+\arcsin \delta$. Thus we may assume that $\psi<90^{\circ}+\operatorname{arc} \sin \delta$ which allows us to choose the segment $A B$ on $s$ so as to satisfy (1.2).

Now we introduce an oblique coordinate system $x y$ so that $P=(0,0), 0=$ $(0,-r), A=(a, 0), B=(-b, 0)$, and consider the locus $l_{a}$ of those points $Q=Q(x, y)$ for which

$$
\overline{O Q}-r=(2 a-x+y) \delta
$$

Similarly, we consider the locus $l_{b}$ of the points $Q$ defined by

$$
\overline{O Q}-r=(2 b+x+y) \delta \text {. }
$$

Let $\omega$ be the angle between the $x$ - and $y$-axis. Then by

$$
\overline{O Q}^{2}=x^{2}+(y+r)^{2}+2 x(y+r) \cos \omega, \quad \cos \omega=(1-a) \delta,
$$

we can write (1.3) and (1.4) in the form

$$
s\left(x^{2}+y^{2}\right)+t x y+u x+v y+w=0
$$


with $s=1-\delta^{2}, t=2(1-a) \delta+2 \delta^{2}, u=2 r(2-a) \delta+4 a \delta^{2}, v=2 r-2 r \delta-4 a \delta^{2}$, $w=-4 r a \delta-4 a^{2} \delta^{2}$ for $l_{a}$ and $s=1-\delta^{2}, t=2(b-1) \delta-2 \delta^{2}, u=-2 r(2-b)-$ $4 b \delta^{2}, v=2 r-2 r \delta-4 b \delta^{2}, w=-4 r b \delta-4 b^{2} \delta^{2}$ for $l_{b}$.

The sort of the conic represented by (1.5) depends on the sign of $s^{2}-\frac{1}{4} t^{2}$. We are concerned with the case $0<a, b<2,0<\delta<1$. Now $\frac{1}{2}|t|<\delta+\delta^{2}$, so that $s-\frac{1}{2}|t|>1-\delta-2 \delta^{2}$ which is positive for $\delta<\frac{1}{2}$. It follows that, for $r>\frac{3}{4}, l_{a}$ and $l_{b}$ are ellipses.

We have $\overline{O A}-r=a \delta<(2 b+a) \delta$ and $\overline{O B}-r=b \delta<(2 a+b) \delta$ showing, on the one hand, that $A$ and $B$ are lying on $l_{a}$ and $l_{b}$, and on the other hand, that they are contained in the elliptical disks bounded by $l_{b}$ and $l_{a}$, respectively.

We still need some information about the point of intersection $C$ of the tangent of $l_{a}$ at $A$ and the tangent of $l_{b}$ at $B$. The derivative $y^{\prime}$ of $y=y(x)$ defined by (1.5) is given by the equation

$$
2 s\left(x+y y^{\prime}\right)+t y+t x y^{\prime}+u+v y^{\prime}=0 .
$$

Writing $y^{\prime}=y_{A}^{\prime}$ at the point $A$ and $y^{\prime}=y_{B}^{\prime}$ at the point $B$, we obtain, by using the respective coefficients,

$$
y_{A}^{\prime}=-\frac{a+r(2-a) \delta+a \delta^{2}}{r+\left(a-a^{2}-r\right) \delta-a \delta^{2}}, \quad y_{B}^{\prime}=\frac{b+r(2-b) \delta+b \delta^{2}}{r+\left(b-b^{2}-r\right) \delta-b \delta^{2}} .
$$

For $0 \leq a, b \leq 2$ the denominators attain their minimum for $a=2$ and $b=2$. Since the numerators are obviously positive, we have $y_{A}^{\prime}<0$ and $y_{B}^{\prime}>0$, provided that $r(1-\delta)-2(a+\delta) \delta>0$. Since this inequality is satisfied for $r \geq 2$ (even for $r>1.33), C$ is in the intersection of the half-plane $y>0$ and the parallel strip $-b<x<a$.

We continue to show that, for $r \geq 2$, the angles $\alpha$ and $\beta$ of the triangle $A B C$ at $A$ and $B$ are less than $60^{\circ}$. Since changing the role of $a$ and $b$ changes the role of $\alpha$ and $\beta$ simultaneously, it is enough to investigate the angle $\alpha$.

We have

$$
-y_{A}^{\prime}=-\frac{d y}{d x}=\frac{\sin \alpha}{\sin (\omega+\alpha)}=\frac{\tan \alpha}{\sin \omega+\cos \omega \tan \alpha}
$$

whence

$$
\tan \alpha=\frac{-y_{A}^{\prime} \sin \omega}{1+y_{A}^{\prime} \cos \omega}
$$

Using the inequality $r \delta<\frac{1}{2}$ and writing $\delta_{0}=\delta\left(r_{0}\right)$ it is easy to check that, for $r \geq r_{0}, 0 \leq a \leq 2$, we have

$$
-y_{A}^{\prime}<\frac{2+2 \delta_{0}^{2}}{r-0,5-2\left(\delta_{0}+\delta_{0}^{2}\right)}=m(r)
$$


Since $m(r)$ is a decreasing function,

$$
\tan \alpha<\frac{m\left(r_{0}\right)}{1-m\left(r_{0}\right) \delta_{0}}
$$

This yields, for $r_{0}=2.6, \alpha<58.4^{\circ}$. For $2 \leq r<2.6$ numerical computations show that, for a given $r, \alpha$ is an increasing function of $a$ and, for $a=2, \alpha$ is a decreasing function of $r$. The maximum of $\alpha$, i.e., its value for $a=r=2$ is approximately $54.3^{\circ}$.

Now we may assume that neither $A$ nor $B$ are on bd $d$ since these points would satisfy condition (1.1) imposed on the point $P^{\prime}$. Since the diameter of $d$ was supposed to be at least 2 a part of bd $d$ lies outside the triangle $A B C$. Therefore bd $d$ intersects either $l_{a}$ or $l_{b}$ or both. Let bd $d$ intersect $l_{a}$. Proceeding from $P$ on bd $d$ in positive direction, let $P^{\prime}\left(x^{\prime}, y^{\prime}\right)$ be the first point of intersection. We assume that the $y$-coordinate of any point of the arc $P P^{\prime}$ is at most $y^{\prime}$. Then

$$
\Delta r=\overline{O P}-r=(2 a-x+y) \delta>\Delta \lambda \cdot \delta .
$$

Assuming on the other hand that the arc $P P^{\prime}$ contains a point whose $y$-coordinate is greater than $y^{\prime}$, then bd $d$ cannot intersect the side $A C$ of the triangle $A B C$. Therefore it must intersect the side $B C$, and consequently $l_{b}$. Now proceeding from $P$ on bd $d$ in negative direction, the first point at which we reach $l_{b}$ satisfies (1.1).

Now we have proved (1.1) if the respective point $P^{\prime}$ is on the departure side of $d$. In the opposite case we move $P^{\prime}$ on bd $d$ so as to increase the distance $O P^{\prime}$ until the line $O P^{\prime}$ touches $d$. We claim that during this operation $\Delta r / \Delta \lambda$ increases. Changing our notation we write $P_{0}$ and $P_{1}$ for the original points $P$ and $P^{\prime}$, and $P$ for a variable point on the arrival side of $d$ beyond $P_{1}$. Writing furthermore $\overline{O P}=r$ and $\sigma$ for the length of the arc $P_{0} P$, we have $d r / d \sigma=\cos \varepsilon$, where $\varepsilon$ is the angle made by the directed line $O P$ and the instantaneous direction in which $P$ is moving. Since $\varepsilon$ is a decreasing function of $\sigma, r$ is a convex function of $\sigma$. Therefore, in accordance with our statement,

$$
\frac{r-r_{0}}{\sigma}>\frac{r_{1}-r_{0}}{\sigma_{1}}>\delta\left(r_{0}\right)
$$

This completes the proof of the lemma.

Now our strategy of flight from $O$ is as follows. First we travel from $O$ to a point $P_{1}$ of distance 2 from $O$. We proceed on the half-line $O P_{1}$ until we get to a point $P_{2}$ on the arrival side of a disk (which may coincide with $P_{1}$ ). We continue our way on the boundary of this disk to the point $P_{3}$ on its departure side satisfying (1.1), proceed on the half-line $O P_{3}$ to the next disk, and so on.

In order to get an upper bound for the total length $\lambda_{n}$ of our path from $O$ to 
$P_{n}$ in terms of $r_{n}=\overline{O P}_{n}$ we write (1.1) in the form

$$
\frac{\Delta \lambda}{\Delta r} \leq \sqrt{1+r^{2}}+r
$$

Using the inequality $\sqrt{1+r^{2}}<r+1 / 2 r$ and writing $f(r)=2 r+1 / 2 r$, we have $\Delta \lambda<f(r) \Delta r$. For a straight segment of the path $\lambda_{2 i}-\lambda_{2 i-1}=r_{2 i}-r_{2 i-1}$, we have, for all positive integers $i$,

$$
\lambda_{i+1}-\lambda_{i}<f\left(r_{i}\right)\left(r_{i+1}-r_{i}\right)
$$

Observing that, for $r>\frac{1}{2}, f(r)$ is an increasing function, we have

$$
\lambda_{n}-\lambda_{1}<\sum_{i=1}^{n-1} f\left(r_{i}\right)\left(r_{i+1}-r_{i}\right)<\int_{2}^{r_{n}} f(r) d r=r_{n}^{2}+\frac{1}{2} \ln r_{n}-4-\frac{1}{2} \ln 2 .
$$

This inequality does not hold generally by replacing $\lambda_{n}$ and $r_{n}$ by the respective data $\lambda$ and $r$ associated with an arbitrary point of the path constructed above because, during evading a disk, $r$ first increases slowly or definitely decreases. Let $P_{n}$ be on the arrival side of a disk $d$ we want to evade. Let $A B$ be a segment of length 2 satisfying (1.2) with $\overline{P_{n} A}=a, \overline{P_{n} B}=b$, and such that $A B$ is a support line of $d$. Let the circle with center $O$ and radius $r$, such that $r_{n}<r \leq \overline{O B}$, intersect the segment $P_{n} B$ at $G$ and bd $d$ in a point $P$ on the arrival side of $d$, such that the line $O P$ intersects the segment $P_{n} B$ in a point $H$. Let $\sigma$ be the length of the arc $P_{n} P$. Then $\sigma \leq P_{n} H+H P \leq P_{n} H+H G=P_{n} G$ with equality if $P$ coincides with $G$. On the other hand, for a given $r, \overline{P_{n} G}=\sigma_{n}$ attains its maximum in the limiting case as $a \rightarrow 0$. For $a=0$ we have

$$
r^{2}=r_{n}^{2}+\sigma_{n}^{2}-2 r_{n} \sigma_{n} \delta_{n}, \quad \delta_{n}=\delta\left(r_{n}\right)
$$

whence $\sigma_{n}=r_{n} \delta_{n}+\sqrt{r_{n}^{2} \delta_{n}^{2}+r^{2}-r_{n}^{2}}$. It follows that during evading $d$ we have

$$
\lambda<g(r)=\lambda_{n}+r_{n} \delta_{n}+\sqrt{r_{n}^{2} \delta_{n}^{2}+r^{2}-r_{n}^{2}}
$$

We write $h(r)=r^{2}+\frac{1}{2} \ln r-4-\frac{1}{2} \ln 2$ and consider the difference $D(r)=$ $g(r)-h(r)$. Since $D(r)$ is concave and

$$
D^{\prime}\left(r_{n}\right)=\frac{1}{\delta_{n}}-2 r_{n}-\frac{1}{2 r_{n}}=\sqrt{1+r_{n}^{2}}-r_{n}-\frac{1}{2 r_{n}}<0
$$

we have, for $r \geq r_{n}$,

$$
D(r) \leq D\left(r_{n}\right)=\lambda_{n}+2 r_{n} \delta_{n}-h\left(r_{n}\right)<\lambda_{1}+2 r_{n} \delta_{n}<\lambda_{1}+1
$$

and consequently $\lambda<h(r)+1$.

This ends the proof of (1) with the constant $c=\Lambda(2)-4-\frac{1}{2} \ln 2+1$. 


\section{Lower Bound}

We construct a packing showing that, for $r \geq \cot (\pi / 9) \approx 2.75$,

$$
\Lambda(r) \geq r^{2}-\left(\frac{\pi}{3}+\frac{3}{\pi}\right) r+3.6
$$

Let $\ldots, P_{n}^{-2}, P_{n}^{-1}, P_{n}^{0}, P_{n}^{1}, P_{n}^{2}, \ldots$ be regular $n$-gons centered at $O, P_{n}^{i}$ being spanned by the side-midpoints of $P_{n}^{i+1}$. These $n$-gons divide the plane into triangles. Let the inradius of $P_{n}^{0}$ be $R_{n}=\cot (\pi / n)$. Then the diameter of each triangle in the exterior of $P_{n}^{0}$ is greater than or equal to 2 .

Let $r_{n}^{k}$ be the circular ring bounded by the incircles of $P_{n}^{0}$ and $P_{n+k}^{0}$. A rather "good" packing (a packing impeding the flight from $O$ ) is obtained by considering in each ring $r_{n}^{1}$ the triangles generated by the $n$-gons. However, here the flight from $O$ is facilitated by the fact that at each new ring $r_{n}^{1}$ we can start from a point which is generally near to a vertex of $P_{n}^{0}$ avoiding hereby a road section of length 1 along a half-side of $P_{n}^{0}$. Therefore it is convenient to pack two or more consecutive rings with similar triangles. We investigate the packing of triangles in $r_{n}^{3}$ generated by the $n$-gons for $n=6,9, \ldots$ completed by the packing of $P_{6}^{0}$ by triangles and pentagons exhibited in Fig. 1.

Let $P_{n}^{k}$ be the outermost $n$-gon in $r_{n}^{3}$, i.e., the $n$-gon with $k=k(n)$ given by

$$
R_{n} q^{k} \leq R_{n+3}<R_{n} q^{k+1}, \quad q=\frac{1}{\cos (\pi / n)}
$$

Let $\lambda$ be the distance covered by a point $P$ during its flight from $O$ in this packing. Writing $\lambda=\lambda_{n}^{l}$ if $P$ is in a vertex of $P_{n}^{l}(n=6,9, \ldots)$, we have

$$
1+\lambda_{n}^{k}-\lambda_{n}^{0}=1+q+q^{2}+\cdots+q^{k}=\frac{q^{k+1}-1}{q-1}>\frac{\left(R_{n+3}-R_{n}\right) \sin (\pi / n)}{1-\cos (\pi / n)} .
$$

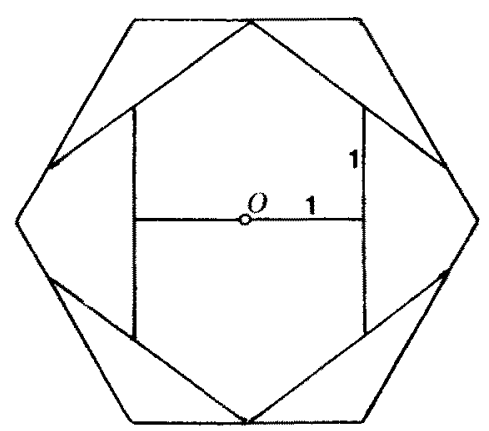

Fig. 1 
In view of

$$
\cot x=\frac{1}{x}-\frac{x}{3}-\cdots, \quad 0<x<\frac{\pi}{2}
$$

and

$$
\frac{\sin x}{1-\cos x}>\frac{x-x^{3} / 6}{x^{2} / 2}=\frac{2}{x}-\frac{x}{3}, \quad 0<x<\frac{\pi}{2}
$$

then

$$
1+\lambda_{n}^{k}-\lambda_{n}^{0}>\left(\frac{3}{\pi}+\frac{\pi}{n(n+3)}\right)\left(\frac{2 n}{\pi}-\frac{\pi}{3 n}\right)=\frac{6 n}{\pi^{2}}+\frac{1}{n(n+3)}\left(n-3-\frac{\pi^{2}}{3 n}\right)
$$

and, by our stipulation that $n \geq 6$,

$$
\lambda_{n}^{k}-\lambda_{n}^{0}>\frac{6 n}{\pi^{2}}-1
$$

This implies, because $R_{n}<n / \pi$ and

$$
\begin{gathered}
\lambda_{6}^{0}=2+\sqrt{32-18 \sqrt{3}}+\sqrt{28-16 \sqrt{3}}>3.44 \\
\Lambda\left(R_{3 n}\right) \geq \lambda_{3 n-3}^{\lambda}>\lambda_{6}^{0}+\lambda_{6}^{k}-\lambda_{6}^{0}+\lambda_{9}^{k}-\lambda_{9}^{0}+\cdots+\lambda_{3 n-3}^{k}-\lambda_{3 n-3}^{0} \\
>\lambda_{6}^{0}+\frac{6}{\pi^{2}}(6+9+\cdots+3 n-3)-(n-2) \\
=\frac{9}{\pi^{2}} n^{2}\left(\frac{9}{\pi^{2}}+1\right) n+2\left(1-\frac{9}{\pi^{2}}\right)+\lambda_{6}^{0}>R_{n}^{2}-\left(\frac{\pi}{3}+\frac{3}{\pi}\right) R_{n}+3.6
\end{gathered}
$$

proving (2) for values of $r$ of the form $r=R_{3 n}=\cot (\pi / 3 n), n=3,4, \ldots$.

If the fleeing point $P$ is in a vertex of $P_{n}^{l}$, then $\overline{O P}=r=R_{n} q^{l}$. Therefore, for $l=1,2, \ldots, k$,

$$
\lambda_{n}^{l}=\lambda_{n}^{0}+q \frac{q^{l}-1}{q-1}=\lambda_{n}^{0}+\frac{q}{q-1}\left(\frac{r}{R_{n}}-1\right)
$$

is in a linear relation with the pertaining value of $r$. Since, on the other hand, in $\left(\lambda_{n}^{l}, \lambda_{n}^{l+1}\right), \lambda=\lambda_{n}^{l}+\sqrt{r^{2}-R_{n}^{2} q^{2 l+2}}$ is a concave function of $r$, we have, in the whole interval $\left(\lambda_{n}^{0}, \lambda_{n}^{k}\right)$,

$$
\lambda \geq \lambda_{n}^{0}+\frac{q}{q-1}\left(\frac{r}{R_{n}}-1\right)
$$

which is obviously greater than the (convex) lower bound of $\Lambda(r)$ given in (2). 


\section{Remarks}

Let $S T U$ and $S V U$ be triangles defined by $\overline{S U} \leq 2$ and $\overline{S T}=\overline{T U}=\overline{U V}=\overline{V S}=2$. Let $O$ be the center of the quadrangle $S T U V$. We consider two further triangles which arise from the previous ones by a rotation about $O$ through $90^{\circ}$ followed by a translation through $\overline{O S}$ and $\overline{O U}$, respectively. These triangles form a packing which shows that, for $r \leq \sqrt{2}$, we have $\Lambda(r) \geq \sqrt{2} r$. It seems to be very likely that, in this inequality, equality holds suggesting that a universely best packing, in which $\Lambda(r)$ is attained for all values of $r$, does not exist.

Suppose that whenever the point $P$ has evaded a disk we remove all disks and throw a new obstacle in its way. In the family of disks under consideration the most efficient obstacle seems to be a "bar" of length 2 put orthogonally to $O P$ with its center at $P$. This suggests a sharper upper bound for $\Lambda(r)$ than $(1): \Lambda(r) \leq r^{2}$ for $r^{2}=2,3, \ldots$ and

$$
\Lambda(r) \leq r^{2}+\frac{1}{4}
$$

for any value of $r \geq \sqrt{2}$.

Among the numerous problems of estimating $\Lambda(F, r)$ for various families of disks, special attention is due to disks with prescribed quotient of their width $w$ and diameter $d$. More generally, we consider the family $F_{p}$ of disks with $w / d \geq p$. For the triangles in the packing generated by $n$-gons in Section 2 we have $w / d=$ $\frac{1}{2} \tan (\pi / n)$. In this packing the length of a path leading from a vertex of $P_{n}^{0}$ to $O$ is given by

$$
r \sin \frac{\pi}{n}\left(1+q+q^{2}+\cdots\right)=r \frac{\sin (\pi / n)}{1-\cos (\pi / n)},
$$

where $r$ is the circumradius of $P_{n}^{0}$. Is it true that, for $p=\frac{1}{2} \tan (\pi / n)$,

$$
\Lambda\left(F_{p}, r\right)=\frac{\sin (\pi / n)}{1-\cos (\pi / n)} r ?
$$

What can be said for other values of $p$ ? In particular, what can be said for $p=1$ ? A construction by Hortobágyi [1] implies that

$$
\Lambda\left(F_{1}, r\right) \geq \frac{8 \pi / 3-4\left(\arcsin \frac{1}{4}+\arcsin \frac{3}{4}\right)}{\sqrt{15}+\sqrt{7}-\sqrt{12}} r>1.3 r .
$$

About other results concerning shortest paths in packings of disks we refer to Section 2.3 of the survey paper [2] and to [3]-[5].

I am grateful to Wlodek Kuperberg for the following comment. "The upper bound (1) obtained for the case of planar disks $D$ with diam $D \geq 2$ applies directly, as a corollary, to the case of $d$-dimensional convex domains $K$ with diam $K \geq 4$. Perhaps, with some elaboration on the proof, this change of the diameter 
assumption might prove unnecessary. In any case, if the approach to the problem in dimension 2 could be successfully transferred to dimension 3 by means of an analogous lemma, then it would automatically produce the same upper bound for all $d \geq 3$. On the other hand, obtaining a good lower bound for $\Lambda(r)$ in dimensions $d \geq 3$ might require some essential modification of the construction."

\section{References}

1. I. Hortobágyi, Ober die Durchlässigkeit einer aus Scheiben konstanter Breite bestehenden Schicht. Studia Sci. Math. Hungar. 11 (1976), 383-387.

2. G. Fejes Toth, New results in the theory of packing and covering. In: Convexity and Its Applications, edited by P. M. Gruber and J. M. Wills. Birkhäuser, Basel, 1983.

3. A. Florian and H. Groemer, Two remarks on the permeability of layers of convex bodies. Studia Sci. Math. Hungar. 20 (1985), 259-265.

4. J. S. B. Mitchell and C. H. Papadimitriou, Planning shortest paths. Proc. SIAM Conference on Geometric Modeling and Robotics, Albany, NY, 1985.

5. E. Arkin, R. Connelly, and J. S. B. Mitchell, On monotone path among obstacles, with applications to planning assemblies. Proc. Fifth Annual ACM Symposium on Computational Geometry, Saarbrücken, June 1989, pp. 334-343.

Received March 10, 1991, and in revised form November 26, 1991. 